\title{
INSOLARTE: breve revisión de proyectos artísticos en una estancia de investigación
}

\author{
INSOLARTE: brief review of artistic projects from a research stay
}

Ana Mercedes VERNIA. Universitat Jaume I (España).vernia@uji.es

\begin{abstract}
Resumen: Actualmente la música, aunque está perdiendo representatividad y presencia en las instituciones educativas, contrariamente, está tomando relevancia en la sociedad, en la calidad de vida de las personas, en la inclusión educativa y social, además se estima que es una potente herramienta de vertebración social, de empleabilidad y sostenibilidad. A través de proyectos artísticos, la música se comporta como un nexo de unión que permite la implementación en diferentes contextos y perfiles, de acciones capaces de potenciar la motivación, la autoestima, así como mejorar las competencias sociales y personales. Entre los problemas y los retos actuales, nos encontramos por una parte con la empleabilidad y su relación con la educación y la formación en los futuros maestros/as, y otra parte la importancia de la agenda 2030 y los 17 Objetivos de Desarrollo Sostenible (ODS). Nuestra propuesta se fundamenta en una estancia de investigación que se realizó en la universidad de Barcelona, a través de una convocatoria lanzada por la Conselleria d'Educació, Investigació, Cultura i Esport - Direcció General d'Universitat, Investigació i Ciència de la Generalitat Valenciana. Entre los principales objetivos, además de la elaboración de un informe sobre los beneficios de los proyectos artísticos, para la transformación social y educativa, tuvimos presente la relación de la música con la formación de los futuros maestros de educación primaria y los beneficios del arte en general y de la música en especial. Para obtener información se realizaron diferentes entrevistas y se organizaron dos focus group. Hallamos importante información que refuerza la importancia de la formación musical y sus beneficios, así como la relevancia de los proyectos artísticos en la sociedad, sin ninguna discriminación o exclusión. También reflexionamos sobre el impacto económico de los proyectos artísticos, no solo desde la empleabilidad sino también de lo que puede suponer su relación con la salud y la calidad de vida.
\end{abstract}

Palabras clave: educación artística, educación cultural, maestros, inclusión social, sostenibilidad 


\begin{abstract}
Currently, although music is losing representation and presence in educational institutions, on the contrary, it is gaining relevance in society, in the quality of life of people, in educational and social inclusion, and it is also considered to be a powerful tool for structuring social, employability and sustainability. Through artistic projects, music acts as a connecting link that allows the implementation in different contexts and profiles of actions capable of enhancing motivation, self-esteem, as well as improving social and personal skills. Among the current problems and challenges, we find, on the one hand, employability and its relationship with education and training for future teachers, and on the other, the importance of the 2030 agenda and the 17 Sustainable Development Goals (SDGs)). Our proposal is based on a research stay that was carried out at the University of Barcelona, through a call launched by the University of Jaume I (Castellón). Among the main objectives, in addition to preparing a report on the benefits of artistic projects for social and educational transformation, we kept in mind the relationship of music with the training of future primary school teachers and the benefits of art in general and music in particular. To obtain information, different interviews were carried out and two focus groups were organized. We found important information that reinforces the importance of musical training and its benefits, as well as the relevance of artistic projects in society, without any discrimination or exclusion. We also reflect on the economic impact of artistic projects, not only from employability but also from what their relationship with health and quality of life may entail.
\end{abstract}

Keywords: arts education, cultural education, teachers, social inclusion, sustainability

\title{
Introducción
}

Este artículo/informe ha sido gracias a la subvención para estancias de personal investigador doctor en centros de investigación radicados fuera de la Comunitat Valenciana convocada por la Conselleria d'Educació, Investigació, Cultura i Esport - Direcció General d'Universitat, Investigació i Ciència de la Generalitat Valenciana. A partir de esta convocatoria se realizó una estancia de investigación en la universidad de Barcelona, en al que se contó con el Dr. Josep Gustems, responsable de la recepción de la estancia de investigación, y distintos profesores colaboradores A partir de ella se elaboró un informe que se facilitó tanto a la Conselleria d'Educació, Investigació, Cultura i Esport. Direcció General d'Universitat, Investigació i Ciència de la Generalitat Valenciana, como a los colaboradores de la Universidad de Barcelona.

Con el objetivo de compartir los resultados y conclusiones de este informe, se elabora el presente artículo, presentando el desarrollo, las actividades, así como los datos utilizados para dicho informe.

En nuestra propuesta, situamos a la música como eje transversal y herramienta para incardinar la propia cultura y el arte de un contexto determinado. Consideramos las canciones, como palabras unidas a las melodías de un pueblo y su repertorio popular, para 
acercar a la sociedad más vulnerable, permitiendo tanto un mejor acceso a la educación, a la alfabetización o "realfabetización" en aquellos casos, que sus realidades obligaron a continuar una educación. También la empleabilidad y la sostenibilidad, entendiendo esta última como parte de bienestar social. Este bienestar social, pasa también por la salud y la calidad de vida, sobre todo en las personas más vulnerables, el acceso al arte es escaso o nulo.

Partimos de las siguientes hipótesis:

H1. La Educación Musical puede ser una importante herramienta de inclusión social y educativa, y que el patrimonio musical (canciones, bailes, vestuario), resulta motivadora para socializar y generar empleabilidad, conectar personas y entornos vulnerables.

H2. La Educación Musical en sus diferentes manifestaciones puede mejorar los entornos sociales y mejorando la calidad de vida y la salud de las personas mayores vulnerables.

H3. La Educación Musical utilizada para proyectos sociales inclusivos, debe partir de profesionales especialistas para garantizar una mejor implementación.

\section{Objetivos}

Para el objetivo general se propone elaborar un informe que recoja ejemplos y modelos de proyectos artísticos de transformación social, así como reflexiones de expertos y especialistas en el campo del arte y la educación.

\section{Objetivos específicos}

- Conocer los efectos reales de la educación musical, para mejorar entornos sociales y conocer su impacto inclusivo.

- Mejora de los entornos sociales desde la educación musical.

- Conocer la respuesta de actividades artísticas realizadas en entornos sociales desfavorable.

- Dibujar un perfil profesional idóneo, para la implementación de proyectos socio inclusivos desde la música.

- Redactar un informe sobre el impacto del proyecto desde su aplicación.

El planteamiento inicial de la estancia tuvo que modificarse, porque la resolución de la convocatoria se retrasó más de lo previsto, por tanto, la temporalidad de la investigación también tuvo que modificarse y consecuentemente las actividades previstas, así como las herramientas que íbamos a utilizar para la gestión y adquisición de la información. 


\section{Marco teórico}

Este trabajo centra su enfoque en el arte y la cultura y como se pueden mejorar los entornos sociales, por eso insistiremos en proyectos donde el arte se convierte en herramienta para la mejora de los entornos sociales desfavorecidos. El Manifesto for a Healthy and Healthcreating Society (Crisp et al., 2016), señala que, para conseguir una ciudad saludable, debe implicarse toda la sociedad, no solo médicos y políticos, también el mundo de la empresa trabajadoras/es, grupos y asociaciones comunitarias, así como profesorado. Partiendo de este compromiso social, nuestro marco teórico se dividirá entre música, cultura y sociedad, por una parte, sostenibilidad y proyectos artísticos por otra parte.

\section{Música, cultura y sociedad}

Terán Cázares et al. (2015) señala que los entornos sociales son diferentes en cada población, atendiendo los hábitos de ahorro y el tiempo de retiro laboral, actitudes hacia el trabajo, costumbres del cuidado a la salud, por eso es importante, según estas autoras, conocer los efectos del entorno sociocultural como información para las expectativas de empleo. En esta línea, Treloyn (2016), manifiesta la importancia del estudio de la música para una mejor comprensión del mundo, la humanidad y sus sociedades, también desde la cognición musical, la musicología o la musicoterapia.

Queensland Conservatorium Research Centre (2009) llevó a cabo el proyecto Sustainable Futures, en el que se encontró que describir cada práctica musical en términos de su contexto, y en relación con la sostenibilidad, facilita nuevos y útiles conocimientos, ya que según se cita en este trabajo, son muchas las iniciativas relacionadas con la sostenibilidad cultural, en las que se ha incidido desde las prácticas musicales como instrumento, en lugar de considerarlas como partes dinámicas de los ecosistemas.

Estrechando lazos con la música, la cultura con la inclusión social, y de acuerdo con Castillo, Sostegno y López-Arostegi (2012), sobre el derecho que tenemos los ciudadanos en el acceso al empleo, vivienda, salud, educación y cultura, entre otros, como el acceso a la información o las redes sociales, también los proyectos artísticos, pueden permitir unos espacios de contacto, de inclusión de cambio de entornos hacia una mejora social, como puede apreciarse en la siguiente figura.

La música es asociativa, emotiva y comunicativa, es capaz de transportar a un lugar, un tiempo, un momento, un recuerdo. Puede conectarse entre nosotros y en nosotros mismos, es casi instintivo, como un latido del corazón. (Morgan, 2018).

Podemos entender, después de conocer el reportaje EducArte: Análisis de la educación artística en Chile. (González y Lizama, 2013), que la educación artística, será un privilegio y para unos pocos, sufriendo una clara exclusión educativa y social. Entre sus aportaciones, este reportaje señala los avances y el retraso de la educación artística en Chile, sufriendo los cambios de leyes, tomando las artes como entretenimiento, incidiendo de esta manera en la reducción de su presencia en la educación obligatoria. 


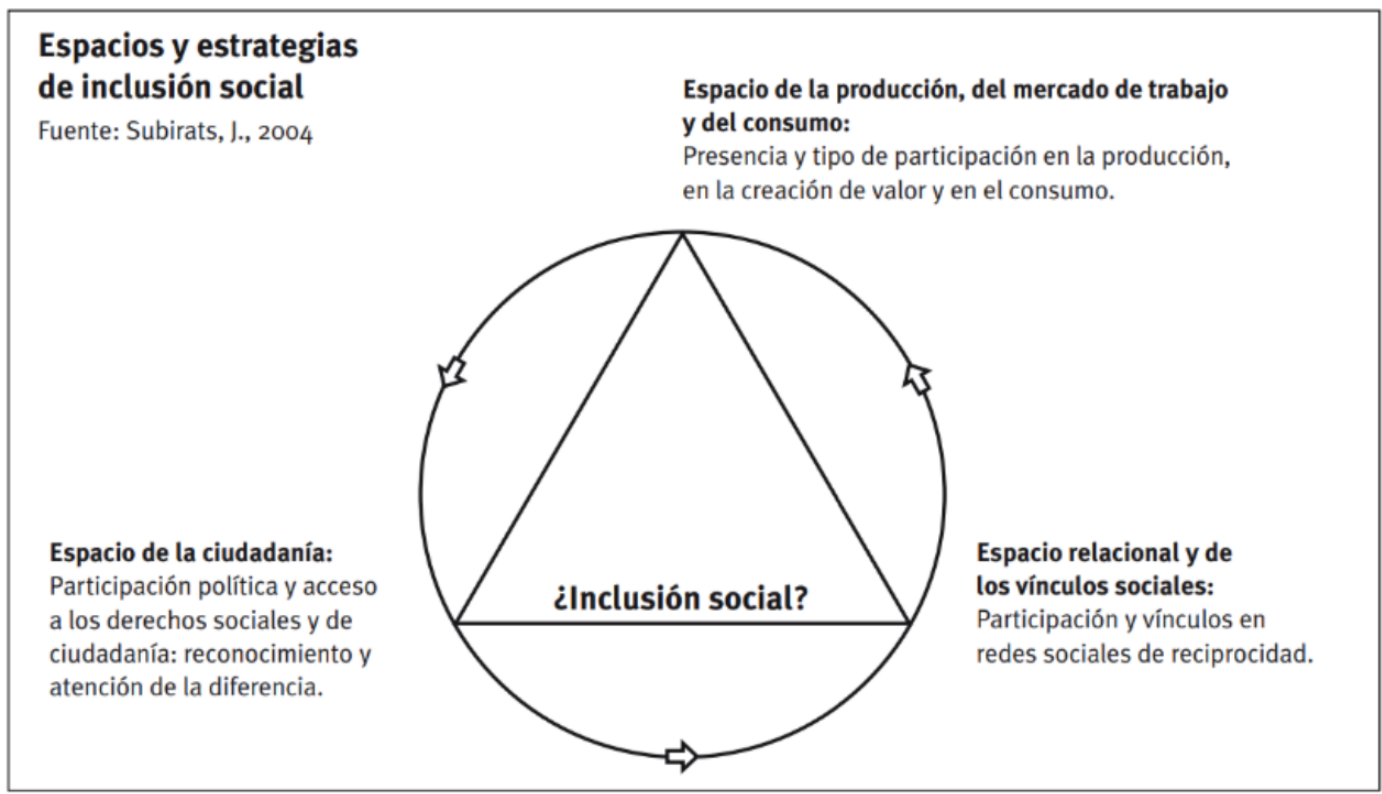

Figura 1. Espacios y estrategias de la inclusión social. Fuente: Subirats (2009).

\section{Sostenibilidad y proyectos artísticos}

En la actualidad, el arte es considerado como una herramienta, para educar, formar, para la mejora de la calidad de vida, como impulso económico. Si tuviéramos que relacionar el arte con los 17 Objetivos de Desarrollo Sostenible 17 (ODS), seguro que encontraríamos relaciones directas o indirectas con todos con la mayoría de ellos. Estos objetivos se adoptaron en la $70^{\mathrm{a}}$ Asamblea General de la ONU (UNESCO, 2015), y ponen de manifiesto las principales preocupaciones mundiales, que manifestaron los jefes de Estado, dirigentes gubernamentales y representantes de alto rango de las Naciones Unidas, así como entidades de la sociedad civil.

En nuestra opinión, estos ODS, encuentran su principal refuerzo en el número 4, que se dirige a la Educación, en su más amplio significado. De acuerdo con las líneas de la UNESCO (2017), que propone una Educación para los ODS, concretamente, unos logros de aprendizaje para fortalecer los sistemas educativos, garantizando una educación inclusiva, equitativa y de calidad. En el marco de la Educación 2030 se promovería oportunidades de aprendizaje para todos y a lo largo de la vida. En este sentido y atendiendo a los del aprendizaje a lo largo de la vida, es necesario atender a todas las edades y perfiles educativos, lo que nos lleva a tener en cuenta las diferentes Ciencias Agógicas, apreciadas en la siguiente tabla, de manera resumida: 
Tabla 1

Ciencias agógicas (Lui Lam Campos, 2018)

\begin{tabular}{|c|c|}
\hline La Paidología & $\begin{array}{l}\text { Estudia la educación de niños en su etapa de preescolar de 3-6 años } \\
\text { de edad. }\end{array}$ \\
\hline La Pedagogía & $\begin{array}{l}\text { Estudia la educación del niño en su etapa de Educación Básica. Es } \\
\text { una ciencia aplicada con características psicosociales que } \\
\text { tiene la educación como principal interés de estudio. }\end{array}$ \\
\hline La Hebegogía & $\begin{array}{l}\text { Estudia la educación del adolescente en su etapa de Educación } \\
\text { Media y Diversificada. }\end{array}$ \\
\hline La Andragogía & Estudia la educación de las personas adultas hasta la madurez. \\
\hline La Gerontogogía & Estudia la educación de adultos en su tercera edad. \\
\hline La Antropogogía & $\begin{array}{l}\text { Es la ciencia y el arte de instruir y educar permanentemente al } \\
\text { hombre, en cualquier período de su desarrollo psico-biológico y en } \\
\text { función de su vida natural, ergológica social. }\end{array}$ \\
\hline La Ergología & $\begin{array}{l}\text { Estudio de la ciencia o filosofía de la ciencia del trabajo, los } \\
\text { presupuestos y condiciones del trabajo, las técnicas, biológica y } \\
\text { mecánica del trabajo, su organización y valoración. }\end{array}$ \\
\hline La Paragogía & Estudio crítico y la práctica de aprendizaje de pares o entre iguales. \\
\hline La Heutagogía & $\begin{array}{l}\text { Comprende al estudiante adulto en su capacidad de aprendizaje } \\
\text { auto-determinado, superando a la andragogía desde el punto de } \\
\text { vista de la autonomía del estudiante adulto. }\end{array}$ \\
\hline
\end{tabular}

\section{Marco Metodológico}

En relación con la investigación social, que es nuestro enfoque, según Canales (2006), se ha de diseñar sus instrumentos y elegir sus estrategias, abandonando los protocolos. En el enfoque dialéctico, este autor señala que los investigadores identifican a los participantes como sujetos y actores en sus relaciones sociales. Enfoque que se relaciona con las premisas de Paulo Freire, centrándose en problemas e interrogaciones de los entornos sociales de los participantes, generando un proceso de educación popular, transformando su realidad. La investigación social, se puede entender como un camino para saber de problemas o necesidades sociales. Nuestra investigación, de carácter sincrónico y descriptivo, busca conocer un problema social en un momento determinado, utilizado la metodología cualitativa, a fin de recoger los datos necesarios, para dar respuesta a las hipótesis, conseguir los objetivos y elaborar el informe que nos permita su aplicación en otro contexto. Dentro de lo que sería la investigación cualitativa, tenemos la vertiente narrativa, dentro del ámbito de las ciencias sociales y humanas. Como dice Blanco (2011), los mismos especialistas aceptan que la investigación narrativa tiene 
muchos puntos en común con otros tipos de investigación de carácter cualitativo, como la autobiografía y relatos de vida, o también la autoetnografía.

Por otra parte, la investigación de carácter biográfico-narrativo posibilita que el sujeto aporta sus propias vivencias, y éstas son el elemento central de las historias de vida que son definidas por Pujadas (1992) como un relato autobiográfico obtenido mediante entrevistas sucesivas en que se muestra el testimonio subjetivo del entrevistado, recogiendo tanto los acontecimientos como las valoraciones que este sujeto hace de su propia existencia. Podemos decir que las historias de vida son una de las herramientas más útiles si queremos entender la vida de las personas posibilitando un punto de vista más amplio y adecuado a la complejidad del ser humano y su mundo de vida (Fernández y Ocando, 2005).

Como indican Bolívar y Segovia (2006, en Huch Aguilar y Reyes Chávez, 2013), la investigación biográfica, especialmente la narrativa, permite que afloran y se desarrollan perfiles que vinculan estrategias cualitativas de investigación a los actores reales de la vida cotidiana. En este caso la narración biográfica ofrece un marco conceptual y metodológico para analizar aspectos esenciales del desarrollo humano y establece sus líneas personales y expectativas de desarrollo.

Dentro de esta línea, nuestra investigación utiliza la perspectiva narrativa y descriptiva, pero desde las experiencias artísticas vividas y por vivir.

\section{Desarrollo de la estancia de investigación/proyecto}

El proyecto se desarrolló con diferentes fases, de implementación, recogida de datos, análisis y elaboración de informes, difusión y prospectiva. Las actividades se llevaron a cabo en el entorno a la Universidad de Barcelona. En la siguiente figura se ha dispuesto algunos de los proyectos artísticos de impacto social, facilitados por los especialistas consultados:

Tabla 2

Proyectos artísticos de impacto social consultados

Esbart Català de Bailan y hacen participar a toda la gente del barrio que quiera. Se Dansaires - $\quad$ puede formar parte del cuerpo de danza a nivel estable o puedes \begin{tabular}{l|l} 
Barcelona & ir a bailar en la sesión semanal que hacen cada viernes y la de las
\end{tabular} fiestas señaladas.

El Tecler - Aula de EL proyecto, AMTP, lo inició el Departamento de Cultura de la Música Tradicional i Generalidad de Cataluña el curso 1992-93 y llevó su gestión hasta Popular - Tarragona el curso 2011-12. Actualmente el proyecto continúa en manos de entidades privadas esparcidas por tres lugares del territorio: Tortosa, Girona y Tarragona. El objetivo es que todo el mundo puede tocar y hacer música tradicional y popular y participar en sus manifestaciones. 


\begin{tabular}{|l|l|}
\hline Ritmo en los barrios & $\begin{array}{l}\text { Organización que trabaja la educación musical con niños de } \\
\text { diferentes barrios de Nicaragua. }\end{array}$ \\
\hline Xamfrà & $\begin{array}{l}\text { De impacto sociales en barrios de Barcelona. Centro de música } \\
\text { y escena del Raval de Barcelona que usa la música, el teatro y la } \\
\text { danza como herramientas de participación e inclusión social. }\end{array}$ \\
\hline Músicas para la vida & $\begin{array}{l}\text { En personas mayores / adultas, para la mejora de la calidad } \\
\text { de vida y la prevención de demencias. Actualmente, este } \\
\text { proyecto se desarrolla en L'Alcora (Castellón), desde el grupo } \\
\text { de investigación Q-HEART (de la Universitat Jaume I. Se ha } \\
\text { iniciado colaboración con médicos psicólogos e informáticos } \\
\text { para desarrollar aplicaciones y conocer el impacto que la música, } \\
\text { practicada de manera actica, ejerce sobre personas mayores. }\end{array}$ \\
\hline Música y palabra & $\begin{array}{l}\text { En personas mayores, para la mejora de la memoria y la calidad } \\
\text { de vida. Se desarrolló en 2014 en la Residencia Azahar del } \\
\text { Mediterráneo en convenio con la Universidad Jaume I. }\end{array}$ \\
\hline Art transforma & $\begin{array}{l}\text { ARTransforma es una entidad sin ánimo de lucro, creada en 2009 } \\
\text { por profesionales de la cultura, del arte y del ámbito social, con el } \\
\text { objetivo de fomentar oportunidades de participación, educación } \\
\text { y profesionalización artística. Para hacer realidad la resolución } \\
\text { 48/96 de la ONU: El derecho a la cultura es un derecho inalienable } \\
\text { a la persona. La igualdad de oportunidades en cuanto al acceso a la } \\
\text { cultura, a las obras de arte, el patrimonio y las prácticas artísticas } \\
\text { es uno de los valores de la identidad. }\end{array}$ \\
\hline $\begin{array}{l}\text { Corals } \\
\text { juvenils i d'adults }\end{array}$ & $\begin{array}{l}\text { Personas de diferentes edades, valores, orientaciones y niveles } \\
\text { musicales y llevando la música en directo en muchos pueblos y } \\
\text { ciudades, permitiendo una experiencia musical tanto al público } \\
\text { como a sus integrantes. }\end{array}$ \\
\hline infantila
\end{tabular}

\section{Herramientas}

En opinión de Granados (2020), los métodos eficaces para investigar suelen presentar un enfoque cualitativo, cuantitativo y mixto, siendo las herramientas más utilizadas la encuesta, cuestionario y entrevista, entre otros. Por su parte, Martínez Miguélez (s.f.) señala que la técnica de focus group es idónea para explorar los conocimientos y experiencias de las personas en contextos de interacción, que posibilitan la discusión y activa a los participantes a comentar y opinar lo que invita a generar una gran riqueza de testimonios.

Para nuestra propuesta escogimos las herramientas siguientes porque, dado el enfoque de la estancia, la temporalización y los recursos de que disponíamos, consideramos que nos aportarían la información necesaria para responder a los objetivos planteados, teniendo en 
cuenta que tuvimos que modificar el cronograma tentativo, reduciéndolo para adaptarnos al nuevo marco temporal.

- Entrevistas

- Cuestionarios

- Focus group

En la siguiente figura podemos observar las sesiones y los objetivos en cada una de los instrumentos utilizadas para la recolección de datos:

Tabla 3

Instrumentos, sesiones y objetivos

\begin{tabular}{|l|l|l|}
\hline Herramientas & Sesiones & Objetivos \\
\hline Cuestionarios & $\begin{array}{l}\text { En diferentes sesiones a lo } \\
\text { largo de la estancia. }\end{array}$ & $\begin{array}{l}\text { Conocer el estado de la cuestión } \\
\text { real y la opinión de los expertos/as }\end{array}$ \\
\hline Entrevistas & Dos sesiones & $\begin{array}{l}\text { Dar una opinión más centraliza- } \\
\text { da sobre un perfil docente y la } \\
\text { repercusión en la formación del } \\
\text { maestro/a }\end{array}$ \\
\hline Focus group & $\begin{array}{l}\text { Diferentes sesiones durante } \\
\text { la estancia, agrupadas en } \\
\text { temáticas vinculadas a los } \\
\text { objetivos }\end{array}$ & $\begin{array}{l}\text { Realizar un debate sobre la } \\
\text { temática de la estancia para la } \\
\text { reflexión y propuesta de opciones } \\
\text { de implementación }\end{array}$ \\
\hline
\end{tabular}

\section{Cronograma tentativo}

El primer cronograma que establecimos en la propuesta, no pudo llevarse a cabo, puesto que la convocatoria se resolvió más tarde de lo previsto, por lo que hubo que planificar las actividades de nuevo, para adaptarlas a la estancia de un mes. Renunciamos a visitar los ejemplos in situ, y pasamos a las entrevistas y focus group, además de compartir los cuestionarios a través de google forms para facilitarnos la temporalidad prevista. 
Tabla 4

Cronograma previsto (no llevado a cabo)

\begin{tabular}{|l|l|l|}
\hline \multicolumn{1}{|c|}{ Acción } & \multicolumn{1}{c|}{ Descripción } & \multicolumn{2}{c|}{ Meses } \\
\hline $\begin{array}{l}\text { Preparación de la } \\
\text { estancia }\end{array}$ & Reuniones y contactos preliminares & \multicolumn{2}{|c|}{} \\
\hline Primera fase & $\begin{array}{l}\text { Implementación de las actividades y segui- } \\
\text { miento }\end{array}$ & \\
\hline Segunda fase & Recogida de datos & \\
\hline Tercera fase & Análisis de los resultados \\
\hline Cuarta fase & Elaboración de conclusiones e informe & \\
\hline
\end{tabular}

Para la cuarta fase, difusión del informe, estamos en el proceso, también esperamos presentar parte del proyecto en un congreso de educación que se llevará a cabo a finales del año en curso (noviembre de 2020).

Tabla 5

Cronograma realizado

\begin{tabular}{|c|c|c|c|c|}
\hline \multirow[t]{2}{*}{ Acción } & \multirow[t]{2}{*}{ Descripción } & \multicolumn{3}{|l|}{ Semanas } \\
\hline & & 2 & 3 & 4 \\
\hline $\begin{array}{l}\text { Preparación de la } \\
\text { estancia }\end{array}$ & $\begin{array}{l}\text { Reuniones y contactos } \\
\text { preliminares }\end{array}$ & & & \\
\hline Primera fase & $\begin{array}{l}\text { Implementación de las } \\
\text { actividades y seguimiento }\end{array}$ & & & \\
\hline \multirow[t]{2}{*}{ Segunda fase } & Recogida de datos & & & \\
\hline & Análisis de los resultados & & & \\
\hline Tercera fase & $\begin{array}{l}\text { Elaboración de } \\
\text { conclusiones e informe }\end{array}$ & & & \\
\hline Cuarta fase & Difusión & & & \\
\hline
\end{tabular}

\section{Resultados}

Los resultados responden a nuestros planteamientos, avalando la relevancia de los proyectos artísticos en la sociedad. La encuesta realizada sobre los entornos sociales y los proyectos artísticos nos aporta información a tener en cuenta, también para la mejora de la mayoría de los Objetivos de Desarrollo Sostenible. 
En la siguiente tabla hemos extraído una pequeña muestra de la información obtenida en las entrevistas.

Tabla 6

Mejora de los entornos sociales desde las artes

\begin{tabular}{|c|c|}
\hline Pregunta & Respuestas \\
\hline $\begin{array}{l}\text { 1. ¿Qué efectos consideras } \\
\text { que produce el arte en general } \\
\text { y la música en especial, en } \\
\text { las personas mayores? }\end{array}$ & $\begin{array}{l}\text { Especialmente, desde las canciones, recuperación de } \\
\text { la memoria. Los efectos que produce el contacto con } \\
\text { el arte en general y la música en particular en personas } \\
\text { mayores es el continuo desarrollo de la propia persona } \\
\text { a varios niveles. Este desarrollo está relacionado con el } \\
\text { desarrollo integral de la persona que engloba aspectos } \\
\text { emocionales, cognitivos, motrices, personales y sociales. } \\
\text { El arte en general y especialmente la música les ayuda a } \\
\text { nivel cerebral, y emocional, a expresar sus sentimientos, } \\
\text { sus emociones, es también un vinculador social, depende } \\
\text { como se utilice puede ser una terapia importante, los hace } \\
\text { vivir mejor. }\end{array}$ \\
\hline 2. Y en jóvenes y adultos? & $\begin{array}{l}\text { Al Margen de la empleabilidad, autoestima, potencia } \\
\text { diferentes capacidades relacionadas con el currículooficial. } \\
\text { Estar activo física y psicológicamente. Sociabilización, } \\
\text { cultura y educación. En jóvenes y adultos, el arte en } \\
\text { general ayuda a nivel emocional y cerebral igualmente. } \\
\text { Una manera de canalizar especialmente en los jóvenes las } \\
\text { emociones negativas, abrirse a la expresión y en la alegría } \\
\text { de vivir. }\end{array}$ \\
\hline $\begin{array}{l}\text { 3. ¿Cómo se podrían mejorar } \\
\text { la calidad de los entornos } \\
\text { educativos y sociales desde } \\
\text { la música y el arte? }\end{array}$ & $\begin{array}{l}\text { A través de proyectos que conectan la sociedad, la } \\
\text { educación y las artes, también los museos y auditorios. } \\
\text { Desde repertorios populares. Abrir la música y las artes } \\
\text { a los entornos de los pueblos. Mayor presencia en los } \\
\text { centros educativos. Arte como vínculo cultural y social. }\end{array}$ \\
\hline $\begin{array}{l}\text { 4. ¿Qué tipo de actividades } \\
\text { musicales se podrían realizar } \\
\text { con personas con dificultades } \\
\text { de aprendizaje, por sus } \\
\text { entornos desfavorables? }\end{array}$ & $\begin{array}{l}\text { Depende de las dificultades de aprendizaje, pero con } \\
\text { instrumentos musicales de percusión, piano. A través de la } \\
\text { expresión corporal y la voz. Todos tenemos un instrumento } \\
\text { gratuito que lo podemos utilizar desde que nacemos: la } \\
\text { voz. Actividades atencionales, de sensibilización y de } \\
\text { discriminación, rítmicas y motrices. }\end{array}$ \\
\hline $\begin{array}{l}\text { 5. ¿Qué competencias } \\
\text { deberían desarrollar los } \\
\text { proyectos artísticos? }\end{array}$ & $\begin{array}{l}\text { Sociales, personales, emocionales, cognitivas. Escucha } \\
\text { activa, crítica y analítica de la música. Empatía con el } \\
\text { medio, fomento del liderazgo y del trabajo en equipo, } \\
\text { creatividad e innovación, valor añadido en clave social o } \\
\text { económica. Beneficios a nivel cerebral, a nivel emocional, } \\
\text { a nivel de expresión, de sensibilidad y de valores. }\end{array}$ \\
\hline
\end{tabular}


6. Participas o has participado en algún proyecto artístico de inclusión? Explica brevemente
En entornos desfavorables, desde la música, la palabra, las artes, hacia la inclusión educativa y social en personas mayores. Música tradicional y popular. "Música para Crecer" e inclusión, se hace con adolescentes.

7. Consideras que la Actualmente No. Es necesario repensar el Currículo, las formación de maestros es competencias y las necesidades que la realidad demanda. completa, con respecto a la $\mathrm{La}$ organización curricular continúa primando las música?

8. ¿Podrías hacer unas breves Recomendaciones para mejorar las instituciones educativas, respecto al arte? habilidades instrumentales básicas como son la lengua y las matemáticas. La formación del profesorado es insuficiente. El arte, como práctica y aprendizaje, debe formar parte de los planes educativos como asignatura obligatoria. Sin su presencia significativa la educación estará incompleta. Es necesario que la misma institución educativa crea y apoye proyectos artísticos y que en los propios centros educativos den al arte la importancia y respeto que se merece. Formar a los profesionales de música también con otras disciplinas.

En las diferentes entrevistas se consultaron proyectos de relevancia en la sociedad, tomando el arte para el impacto social, la inclusión social y educativa. En la siguiente tabla recogemos los proyectos que más se destacaron.

Tabla 7

\section{Proyectos de impacto social valorados en la estancia de investigación}

9. Enumera los proyectos de impacto sociales (desde la música y las artes) que congas y haz una breve descripción (no más de 2 líneas)
Xamfrà: de impacto sociales en barrios de Barcelona.

Músicas para la vida: en personas mayores / adultas, para la mejora de la calidad de vida y la prevención de demencias.

Esbart Catalán de Danzantes - Barcelona: Bailan y hacen participar a toda la gente del barrio que quiera.

El Tecler Aula de Música. Música Tradicional y Popular -Tarragona.

EL proyecto, AMTP, lo inició el Departamento de Cultura de la Generalidad de Cataluña el curso 1992-93 y llevó su gestión hasta el curso 2011-12.

Ritmo en los barrios, organización que trabaja la educación musical con niños de diferentes barrios de Nicaragua.

L'Art del Cor, proyecto de autogestión (teórico, práctica y económica), dignidad y participación, por el colectivo de personas mayores, supervisado por un profesional cualificado en Musicoterapia. 


\begin{tabular}{|l|l|}
\hline Art transforma. \\
Música y palabra: en personas mayores, para la mejora de \\
la memoria y la calidad de vida.
\end{tabular}

De los focus groups, se ha obtenido la información que podemos apreciar en la siguiente tabla:

Tabla 8

Debate y reflexiones de los Focus Group

\begin{tabular}{|l|l|}
\hline $\begin{array}{l}\text { La importancia de la música } \\
\text { en la sociedad. }\end{array}$ & $\begin{array}{l}\text { Es fundamental para la sociedad, pero no se le da el } \\
\text { suficiente soporte, aunque la gente tiene la necesidad } \\
\text { de utilizarla. Cuando un país entra en crisis, recortan en } \\
\text { educación musical, cuando lo que deberían hacer es lo } \\
\text { contrario. Cuando más sufre la gente, más necesita de } \\
\text { la música. A nivel social, la música es cohesionadora, ha } \\
\text { estado presente en todas las culturas, desde la antigüedad } \\
\text { hasta la actualidad, por necesidad de expresión. Forma } \\
\text { parte de la vida, de la sociedad. Es una necesidad, que }\end{array}$ \\
también infiere en la salud. En la música caben todos los \\
perfiles de la sociedad.
\end{tabular}

1 Combinación de habilidades interpersonales, sociales, de comunicación, rasgos de la personalidad, actitudes, atributos profesionales, inteligencia social e inteligencia emocional. 


\begin{tabular}{|l|l|}
\hline $\begin{array}{l}\text { Los beneficios de los } \\
\text { proyectos artísticos para } \\
\text { la inclusión (social y } \\
\text { educativa). }\end{array}$ & $\begin{array}{l}\text { Todas las formas de razonamiento tienen una conexión } \\
\text { directa con el proceso de un músico. La música facilita } \\
\text { los procesos complejos cerebrales y te empodera para } \\
\text { superar situaciones complicadas. Facilita la inclusión y } \\
\text { la alfabetización. El pensamiento divergente se enriquece } \\
\text { de las posibilidades que ofrece la creatividad musical, y } \\
\text { el arte. Desde los proyectos artísticos se puede hacer un } \\
\text { trabajo educativo. }\end{array}$ \\
\hline $\begin{array}{l}\text { La formación docente } \\
\text { para la implementación de } \\
\text { proyectos. }\end{array}$ & $\begin{array}{l}\text { El futuro maestro/a de primaria, llega con una mala } \\
\text { predisposición a la formación musical, posiblemente } \\
\text { porque no ha tenido una experiencia positiva en su } \\
\text { formación como alumno/a. La música hoy debe alejarse } \\
\text { de los virtuosismos para acercarse a todas las personas. Se } \\
\text { aprende música "haciendo música". Es necesaria también } \\
\text { la formación en gestión, en psicología y pedagogía. }\end{array}$ \\
\hline $\begin{array}{l}\text { Las responsabilidades éticas } \\
\text { en los profesionales de la } \\
\text { comunidad educativa }\end{array}$ & $\begin{array}{l}\text { El docente debe dominar la especialidad y enseñarla desde } \\
\text { las competencias musicales. El docente universitario } \\
\text { desconecta de la realidad, lo cual supone un alejamiento } \\
\text { de la realidad en las aulas. Es necesario que el docente } \\
\text { también conecte con la sociedad. }\end{array}$ \\
\hline $\begin{array}{l}\text { ¿Qué competencias, } \\
\text { conocimientos y habilidades } \\
\text { debería tener el docente } \\
\text { ideal para la implementación } \\
\text { de proyectos artísticos } \\
\text { inclusivos/sociales? }\end{array}$ & $\begin{array}{l}\text { La interdisciplinariedad. La música es una disciplina } \\
\text { y como tal, necesita de conocimientos conceptuales, } \\
\text { independientemente de la edad o perfil del alumnado. }\end{array}$ \\
$\begin{array}{l}\text { Las propias competencias que demanda la música } \\
\text { (auditiva, vocal, etc.). Una formación como especialista. } \\
\text { Habilidades sociales, personales, emocionales, empáticas. } \\
\text { Capacidad de adaptación., versátil, especialista de su } \\
\text { materia. Capacidad para implicar a los discentes de su } \\
\text { propia formación. }\end{array}$ \\
\hline
\end{tabular}

\section{Conclusiones}

Consideramos, a partir de la investigación realizada, que el impacto de los proyectos artísticos sobre la sociedad y la calidad de vida de las personas, es importante. También la empleabilidad y la sostenibilidad se ligan a los proyectos artísticos, generando empleo desde la cultura, la educación, las artes y la gestión. Además, incide positivamente en la economía y sostenibilidad de una región o país.

Estimamos que la calidad de vida de las personas supone una reducción en ayudas provenientes de sanidad y servicios sociales. La inversión en los proyectos artísticos para la inclusión social, educativa y calidad de vida, puede reportar beneficios a la sociedad en su conjunto, apostando por ciudades, no solamente sostenibles, sino también, educativas y comprometidas con el desarrollo sostenible, apostando por la calidad de vida, la inclusión y la educación. 
La diversidad cultural y social hoy es un hecho y una realidad que crece con conflictos y oportunidades. En este sentido, los proyectos artísticos son herramientas que permiten procesos de integración e inclusión, posibilitando que diferentes culturas vivan y convivan, aprendan de sus riquezas musicales y artísticas, de sus costumbres y valores, generando espacios para una ciudadanía crítica.

Calibrar el impacto de un proyecto artístico significa atender a un importante número de ciudadanos y ciudadanas de un determinado contexto geográfico y social, sin ningún tipo de discriminación o exclusión. Por tanto, existe una implicación positiva en la mejora de la convivencia, de la calidad educativa y de las responsabilidad social y sostenible.

En cifras o datos económicos, se necesitaría una nueva evaluación que calculara la reducción desde el ámbito de la salud en medicinas, personal sanitario, cuidadores, etc. que los proyectos artísticos aportarían, tales como la mejora de la calidad de vida, desde la psicomotricidad, la autoestima, las relaciones sociales y personales tanto de jóvenes como de adultos, la calidad educativa desde la inclusión y la integración.

Debemos destacar, por las reflexiones de los participantes, que la formación de los maestros y maestras está todavía muy alejada de la calidad y la ética educativa, pues los conocimientos, competencias y habilidades que se consideran adecuadas para ejercer la profesión, todavía no parecen estar adquiridas. En este sentido cabe una reflexión hacia las políticas educativas, para tomar conciencia sobre los beneficios de los proyectos artísticos y el arte dentro y fuera de la escuela. Ante esta situación también es necesaria una actuación directa sobre la sociedad, el arte accesible para todos, no como una disciplina elitista sino como un bien social, y un derecho, tanto para su disfrute como para su aprendizaje.

Como reflexión final, se plantea ampliar este trabajo a todas las artes, pues, aunque este trabajo se ha focalizado principalmente en la educación musical, se es consciente de la importancia de las artes en la educación y en la vida ciudadana, no solo por el significado del arte en sí mismo, sino por todas las posibilidades que brinda al ser humano. En este sentido se plantea un nuevo proyecto, siguiendo el mismo planteamiento, pero ampliando a los y las profesionales de las artes plásticas y visuales, teatro, circo, cine y danza.

\section{Referencias bibliográficas}

Blanco, M. (2011). Investigación narrativa: una forma de generación de conocimientos. Argumentos, 24(67), 135-156. Recuperado de http://www.scielo.org.mx/scielo. php?script=sci_arttext\&pid=S0187-57952011000300007\&lng=es\&tlng=es

Canales, M. (Coord.). (2006). Metodología de investigación social. Introducción a los oficios. Chile: LOM Ediciones.

Castillo, R., Sostegno, R. y López-Arostegi. R. (2012). Arte para la inclusión y la transformación social. 3s_innovación. Recuperado de https://mediacionartistica.files. wordpress.com/2013/05/arte-prara-la-inclusion-bizcaia.pdf 
Crisp, N., Stuckler, D., Horton, R., Adebowale, V., Bailey, S., et al. (2016). Manifesto for a Healthy and Health-creating Society. Londres: The Lancet

Fernández, O. y Ocando, J. (2005). La búsqueda del conocimiento y las historias de vida. Omnia, 11. Venezuela: Universidad de Zulia.

González, G. y Lizama, M. (2013). EducArte: Análisis de la educación artística en Chile. Recuperado de http://artes.uchile.cl/noticias/96376/educarte-analisis-de-la-educacionartistica-en-chile

Granados, R. (2020). Revisión teórica de herramientas metodológicas aplicadas en la investigación criminológica. Derecho y Cambio Social.

Huchim Aguilar, D., Reyes Chávez, R. (2013). La investigación biográfico-narrativa, una alternativa para el estudio de los docentes. Revista Electrónica Actualidades Investigativas en Educación. Recuperado de http://www.redalyc.org/articulo.oa?id=44729878019

Lui Lam Campos, M. (2018). De la pedagogía a la hebegogía en la universidad. Recuperado de https://es.scribd.com/document/396382583/articulo-DE-LA-PEDAGOGI-A-A-LAHEBEGOGI-A-EN-LA-UNIVERSIDAD

Martínez Miguélez, M. (s.f.) Los grupos focales de discusión como método de investigación. Recuperado de http://miguelmartinezm.atspace.com/gruposfocales.html

Morgan, J. (2018). Art and Science. Music lives on: fine tuning the memory. The Lancet. Neurology, 17 (3), 211-212. Recuperado de https://www.sciencedirect.com/science/article/ pii/S147444221730399X

Pujadas, J. (1992) El método biográfico: el uso de las historias de vida en ciencias sociales. Madrid: CIS

Queensland Conservatorium Research Centre (2009). Sustainable futures for music cultures. An ecological approach to intangible cultural heritage. Recuperado de https:// www.griffith.edu.au/data/assets/pdf_file/0030/93198/soundfutures.pdf

Subirats, J. (2009). Cómo ser yo mismo, ser como los demás y sentirme reconocido en mi ser distinto. El reto de la nueva ciudadanía. En VVAA. Eres Igual (p. 14). Madrid: Caja Madrid

Terán Cázares, M.M.; García de la Peña, M. E. y Blanco Jiménez, M. (2015). El entorno social como elemento a considerar para predecir la ciudadanización de los trabajadores dentro de la organización. Innovaciones de Negocios 12(23), 133 -151.

Treloyn, S. (2016). Music in Culture, Music as Culture, Music Interculturally: Reflections on the Development and Challenges of Ethnomusicological Research in Australia. Voices, 16(2) Special Issue on "How Music Can Change Your Life and the World" doi: 10.15845/ voices.v16i2 
UNESCO (2015). La UNESCO y los Objetivos de Desarrollo Sostenible. Recuperado de https://es.unesco.org/sdgs

UNESCO (2017). Educación para los Objetivos de Desarrollo Sostenible Objetivos de aprendizaje. Recuperado de https://unesdoc.unesco.org/ark:/48223/pf0000252423

\section{Agradecimientos}

Al profesorado que ha colaborado de la Universitat de Barcelona y externos: Josep Gustems Carnicer, Eugènia Arús, Maria Fernanda Viñas, Maria Antònia Pujol, Sandra Soler. Y también a Eulàlia Abad (Música per crèixer) 